\title{
Geometry of the Icosikaidigon in Orvieto Cathedral
}

\author{
Josep Lluís i Ginovart ${ }^{1}$ - Albert Samper ${ }^{1}$ • \\ Blas Herrera $^{2} \cdot$ Agustí Costa $^{1} \cdot$ Sergio Coll $^{1}$
}

\begin{abstract}
The rose window on the main façade of Orvieto cathedral is unique because its design is based on a 22 -sided polygon. It is well known that the icosikaidigon cannot be constructed using only a compass and a straightedge. Therefore, using a geometric/statistical approach, we intend to unveil which approximate construction method was used by the sculptor to draw this unique rose window.
\end{abstract}

Keywords Rose window · Icosikaidigon · Orvieto cathedral · Classic Geometry

\section{Introduction}

The rose window on the main façade of Orvieto cathedral was designed by the sculptor Andrea di Cione (c. 1308-1368), who made significant changes to the initial proposal by Lorenzo Maitini (c. 1275-1330) in 1310. Andrea di Cione, also

$\triangle$ Albert Samper

albert.samper@urv.cat

Josep Lluís i Ginovart

josep.lluisg@urv.cat

Blas Herrera

blas.herrera@urv.cat

Agustí Costa

agusti.costa@urv.cat

Sergio Coll

sergio.coll@urv.cat

1 Unitat predepartamental d'Arquitectura, Universitat Rovira i Virgili, Avinguda de la Universitat, 1, 43204 Reus, Spain

2 Departament d'Enginyeria Informàtica i Matemàtiques, Universitat Rovira i Virgili, Avinguda Països Catalans, 26, 43007 Tarragona, Spain 

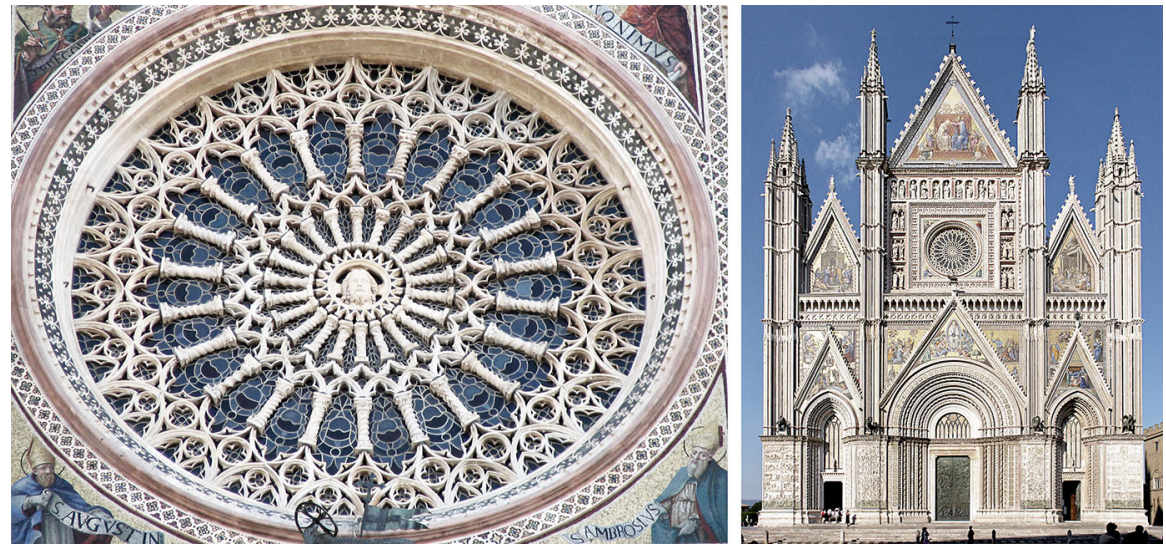

Fig. 1 Main façade of Orvieto cathedral and rose window detail

known as Orcagna, replaced the octagon-based design with a new design based on a 22-sided polygon (Damiani 1991; Bonelli 1951) (Fig. 1). This geometrical base is unusual in Gothic architecture save for a few similar instances such as the rose window of the main façade of Troia cathedral or the rotunda of the church of Neuvy-Saint-Sépulchre in the French Indre department.

\section{Geometric Foundation to the Design of a Rose Window}

"Rose window" is the name given to the most important stained glass window of a Gothic cathedral. This element could have been designed based on the geometrical knowledge of medieval master masons, commonly referred to as geometria fabrorum (Shelby 1965). Another thesis maintains that certain learned geometries were used, in line with similar methods used to build astrolabes, such as De mensura astrolabii (c.1050) by Hernán de Contracto (1013-1050) (Pez 1853). Also, other methods are linked to some Practica Geometriae (1125-1130), such as the one by Hugo de San Víctor (c. 1100-1141) (Baron 1956); the Artis cuiuslibet consummatio (c.1193-c.1220), which is attributed to Gérard de Bruxelles (f.1220) (Victor 1979); or the Practica Geometriae (1346) by Dominicus de Clavasio (+c.1362) (Busard 1965).

Dividing the circle into equal parts is one of the problems that medieval master builders had to face when designing and constructing rose windows. Mostly, these architectural elements were drawn accurately on the basis of 3-, 4-, 5- and 15-sided regular polygons (Fig. 2). The geometric methods for constructing these polygons were known since Euclid (Heath 1908) or Ptolemy (Toomer 1984). Nonetheless, among all the Gothic rose windows, there are a few which cannot be constructed using only a compass and a straightedge, the most important of which is the rose window in Orvieto cathedral. The design of the rose window of Orvieto cathedral is based on a 22-sided polygon. Since the regular 11-sided polygon does not fulfill 

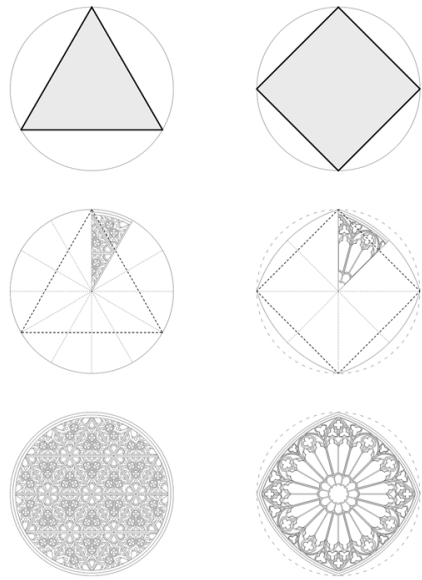
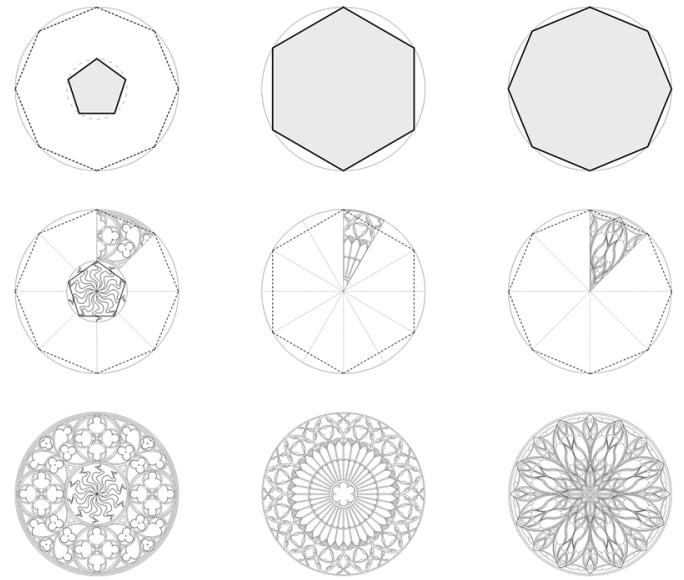

Fig. 2 Designs of the rose windows featured in the cathedrals of Mallorca, Bourges, Milan, Troyes and Amiens

Gauss's Theorem on the regular polygons which can be constructed by Euclid's method, the regular hendecagon cannot be constructed using only a compass and a straightedge. The special case of the icosikaidigon in the Gothic style deserves a careful analysis, which is why in our paper we intend to reveal which approximate geometric method was used by the sculptor to design the 22-sided polygon underlying this rose window.

Our intention is justified, since the actual method used by the sculptor has become blurred with time due to several technical conditions and natural occurrences (error in construction, seismic disturbances, tectonic settlement of the façade, intentional adjustments and tampering, etc.). Figure 3 shows the effects of seismic disturbances and mechanical settlements of the building. Several cracks can be seen, mainly in the central area (Bonelli 1951). Due to all these alterations, determining the geometric method used is not a trivial task, and it cannot be done by mere superposition upon the rose window. As will be described below, in order to solve this problem we need a symbiosis of geometric and statistical techniques. This work will provide statistical results to supplement the epistemological and historical knowledge about Gothic architecture.

\section{Gathering of Information}

Our analysis relies on massive data acquisition techniques by means of terrestrial laser scanning (TLS). This procedure allows for a precise study of the architectural heritage, provides a great geometric definition and makes it possible to measure objects which are inaccessible in the building without having to make contact with them. Besides, it is a very reliable and thoroughly tested technique (Guarnieri et al. 2004). However, it has some limitations regarding the error range for the data acquisition. 


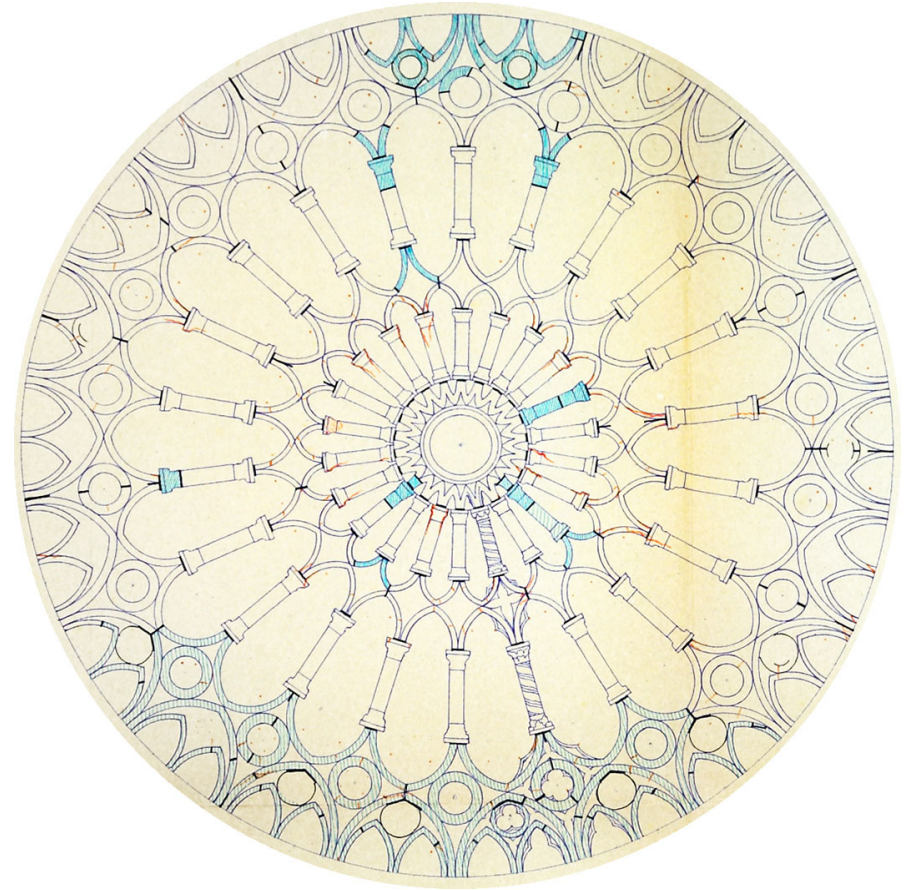

Fig. 3 Document courtesy of the Museum of the Opera del Duomo di Orvieto $\subset$. Archivio dell'Opera del Duomo di Orvieto, Archivio Renato Bonelli, B. 27, fasc. 270

As we have already mentioned above, we will focus our analysis on the outermost crown (of the five crowns which make up the rose window) because, in principle, it is the most rigid one.

The graphical representation of the rose window was made using a 3D laser scanner (Leica ScanStation P20: the maximum instantaneous speed is 1,000,000 points/s; compact model with a dual-axis compensator, precision, range and fieldof-view at topographic level and laser plummet), with an accuracy of less than half a millimetre $(0.0002 \mathrm{~m})$ and from 11 different positions (eight positions at pedestrian level and three positions from the roof of Museum Claudio Faina, right in front of the cathedral) in order to cover the whole façade (Fig. 4). The geometric information of each position was processed with the software Cyclone so as to obtain a complete point cloud of the main façade. This cloud is composed of more than 100 million points, of which more than 10 million points correspond to the rose window (Fig. 5). After assigning coordinates to all the points (Fig. 5), we select the points corresponding to the rose window area and, using the vectorial software AutoCad, we obtain all necessary parameters to make the calculations.

The 22 points for our research have been identified through visual assessment. Among all the topographic points, we have selected the 22 points closest to the vertex of each perimeter arcade, being the spread of these points smaller than $0.002 \mathrm{~m}$. Figure 5 and Table 1 show the set of points that we geometrically and 


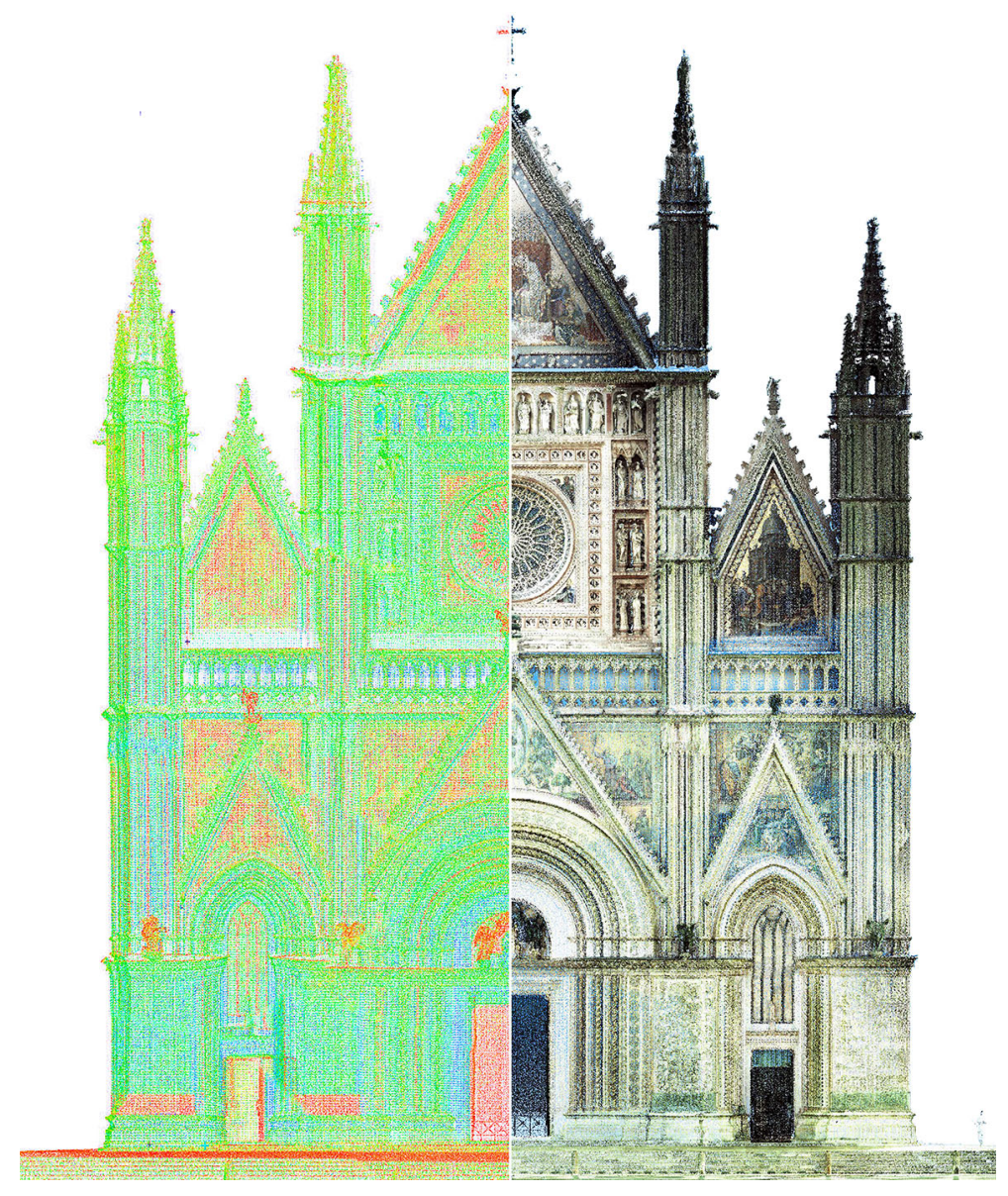

Fig. 4 Points and photographic texturization obtained with the 3D scanner

statistically compare to the approximate methods for constructing an icosikaidigon, as shown in the next section. The accuracy of the data acquisition process-choice of points and computer processing — ranges between 0.003 and $0.004 \mathrm{~m}$. Therefore, the error of this analysis is $1 / 1000$. We consider this to be an acceptable error for a mathematic and statistical processing of this kind.

\section{Approximate Methods for Constructing an Icosikaidigon}

In this section we compile thirteen approximate methods for constructing an icosikaidigon. We have divided them into two subsections. Firstly, we present six methods $\left(M_{1-6}\right)$ which were frequently used in classic and medieval architectures; and secondly, we show seven methods $\left(M_{7-13}\right)$ for which there is no evidence of use in the Middle Ages but are comprised of elemental geometric steps. 

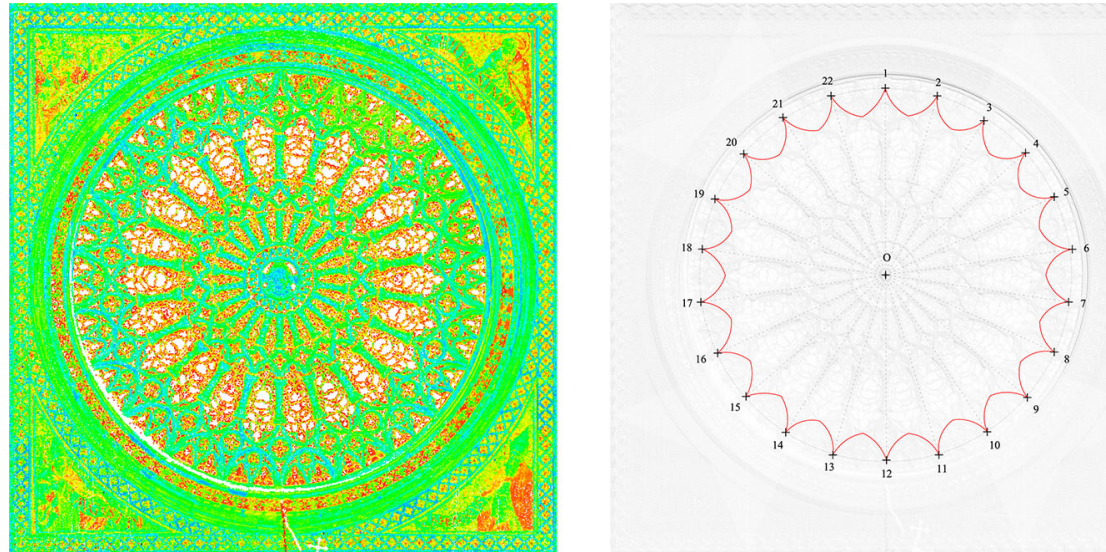

Fig. 5 On the left, detail of the point acquisition. On the right, the 22 vertices $v_{i}$

Table 1 Coordinates of the 22 vertices and side lengths

\begin{tabular}{lll}
\hline$i$ & Coordinates $\left(x_{i}, y_{i}\right)$ of vertices $v_{i}$ & Side lengths $m_{i, i+1}$ \\
\hline 1 & $(3.685,6.211)$ & 0.7037 \\
2 & $(4.381,6.106)$ & 0.7082 \\
3 & $(5.008,5.776)$ & 0.7051 \\
4 & $(5.566,5.346)$ & 0.7017 \\
5 & $(5.950,4.758)$ & 0.7417 \\
6 & $(6.193,4.057)$ & 0.7067 \\
7 & $(6.144,3.352)$ & 0.6957 \\
8 & $(5.951,2.684)$ & 0.7073 \\
9 & $(5.589,2.076)$ & 0.7059 \\
10 & $(5.052,1.618)$ & 0.7168 \\
11 & $(4.405,1.310)$ & 0.7071 \\
12 & $(3.701,1.239)$ & 0.7225 \\
13 & $(2.982,1.308)$ & 0.7007 \\
14 & $(2.349,1.610)$ & 0.7439 \\
15 & $(1.818,2.090)$ & 0.6935 \\
16 & $(1.438,2.670)$ & 0.7167 \\
17 & $(1.212,3.350)$ & 0.7092 \\
18 & $(1.230,4.059)$ & 0.6905 \\
19 & $(1.400,4.729)$ & 0.7155 \\
20 & $(1.787,5.331)$ & 0.7155 \\
21 & $(2.312,5.817)$ & 0.7079 \\
22 & $(2.958,6.107)$ & 0.7352 \\
\hline
\end{tabular}



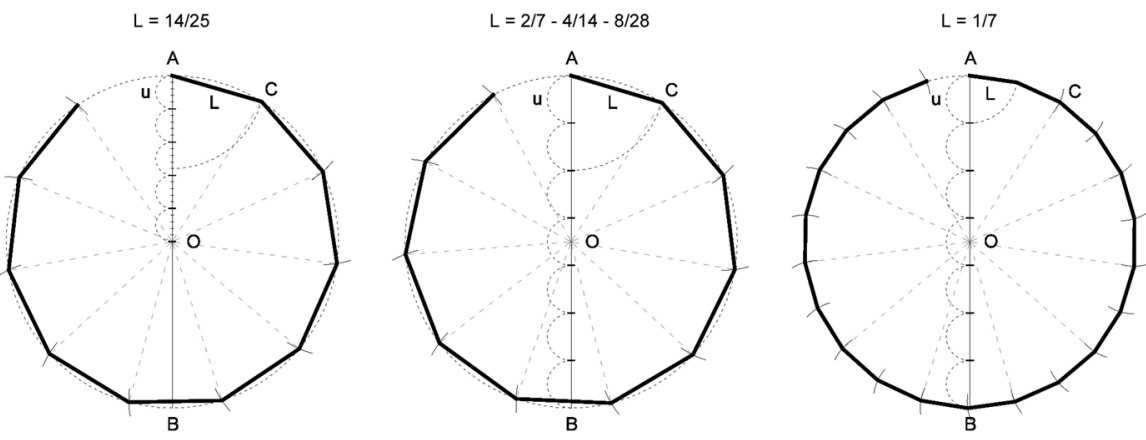

Fig. 6 Left to right: methods $M_{1}, M_{2}$ and $M_{3}$

\section{Methods That We Are Certain Were Known in the Middle Ages}

\section{$M_{1-3}:$ Construction Based on Approximations to Pi}

This first method is based on the approximations to $p i$ which were initially put forward by Archimedes and then by Vitruvius and Ptolemy. This method was used in the design of many classical Greco-Roman buildings and consists of the following steps (Fig. 6): given a circle with centre $O$ and diameter $\overline{A B}$, we divide the diameter into $N$ parts, each of them being the unit $u$. Next, using a compass set with a radius which is $n$ times $u$ and its centre on point $A$, we draw an arc which cuts the given circle at the point $C$. We define $\overline{A C}$ as the segment of the polygon which is inscribed in the given circle. Next, with centre on point $C$ and radius $\overline{A C}$, we draw an arc in order to find another vertex of the polygon. Lastly, similar to the previous step, we find the rest of the vertices. Based on the ratio $\frac{\overline{A B}}{N}$ we can find the approximate side length of a regular polygon.

In the particular case of the icosikaidigon, there are several historical references and examples of architectural elements which were built based on specific ratios. Here are a few examples: according to Thomas Heath (1908), ratios 14/25 and 2/7 were commonly used in classical Greek constructions; Wilson (2000) shows that the circular floor of the mausoleum Le Carceri Vecchie in Capua consists of 22 modules which were laid out based on the ratio 1/7; and finally, with respect to architectural ornamentation, in the city of Tarragona there is a 22-part circular Clypeus from the first century A.D. which includes an image of Jupiter-Ammon and was designed on the basis of the ratio 14/25 (Toldrà et al. 2014).

In this paper we consider the three most common ratios used in architecture for an approximate construction of the icosikaidigon and the hendecagon (Fig. 6). These ratios are: 14/25 $\left(M_{1}\right), 2 / 7\left(M_{2}\right)$ and 1/7 $\left(M_{3}\right)$. The analytical equations of the angles of these methods are shown in "Geometric and Statistical Control", section. 


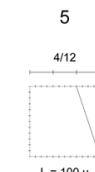

$L=100 u$
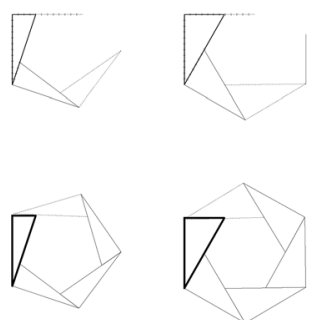

$L^{\prime}=102.6149 u$

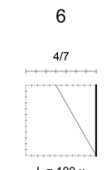

$L=100 u$

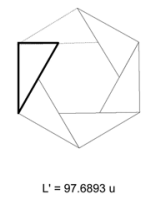

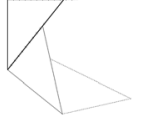
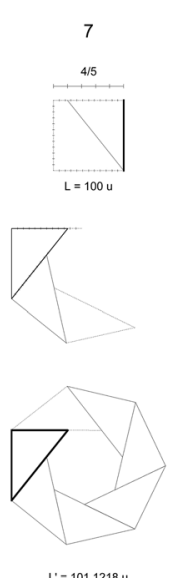

$L^{\prime}=101.1218 u$

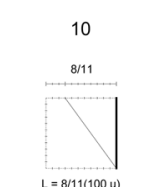

$L=8 / 11(100 u)$
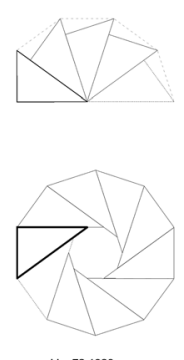

$L^{\prime}=72.1928 \mathrm{u}$

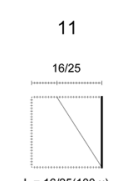

$L=16 / 25(100 u)$
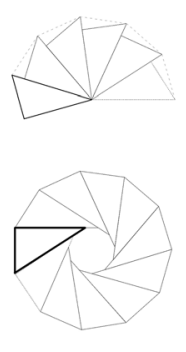

$L^{\prime}=66.2647 u$

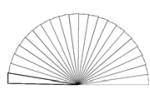

44

$1 / 7$
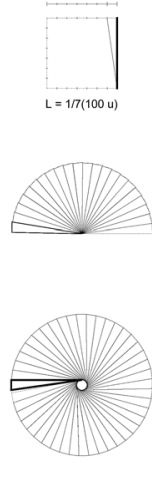

$L^{\prime}=18.2743 u$

Fig. 7 Set-square rotation method. On the right: methods $M_{4}$ and $M_{5}$

\section{$M_{4}, M_{5}$ : Construction by Means of Set-Square Rotation}

Set squares were defined in Plato's Timaeus, where the triangle is considered to be the most important shape in geometry (Lisi 1997). Although classic set squares continued to be used (Recht 1989), a new kind of set square appeared in the thirteenth century. However, throughout history we find variations, such as: canonical set squares $\left(30^{\circ}-60^{\circ}\right)$ by Hugues Libergier (Wu 2000); Pythagorean set squares $(3 \mathrm{u}, 4 \mathrm{u}, 5 \mathrm{u})$ by Niederhaslach; root-five rectangle set squares; decagonal coordinates set squares $\left(36^{\circ}-54^{\circ}\right)$; and lastly, those set squares which are designed based on the golden ratio $\left(31.72^{\circ}-58.20^{\circ}\right)$.

In order to construct a given polygon, the ratio of the set-square legs had to be known (Fig. 7). For instance: ratio 4/12 was used to construct a pentagon; ratio 4/7 was used to construct a hexagon; ratio $4 / 5$ can be used for the approximate construction of a heptagon; ratio $4 / 4$ can be used to construct an octagon; ratio 8/11 was used to construct a decagon, and lastly ratio 16/25 was used for the approximate construction of a hendecagon. Next, using a set square measuring $L$ in length, it could be rotated while a leg was laying on the hypotenuse. The number of movements in a full rotation depended on the set-square proportions and the polygon to be constructed.

However, this method did not offer an exact solution in all cases. In this paper we will consider two ratios which are known to have been used to construct the 11-sided polygon and the 44-sided polygon (divisor and multiple of the icosikaidigon) and have already been applied in other architectural references (Wilson 2000). These ratios are: $16 / 25\left(M_{4}\right)$ and $1 / 7\left(M_{5}\right)$. The analytical equations of the angles of these methods are shown in "Geometric and Statistical Control"' section.

\section{$M_{6}:$ Construction Using an Astrolabe}

The popularity of the astrolabe in the Middle Ages was mainly due to the possibility of measuring angles using trigonometric methods, thus enabling measurement of 
distances in inaccessible areas, height calculations and even deepness deduction. In the astrolabes which were typically made and used during the twelfth to fourteenth centuries, the rim of the matter is graduated with 72 marks, one approximately every $5^{\circ}$ (Eagleton 2007). This geometric construction was obtained by bisecting the $30^{\circ}$ angle and then trisecting the $15^{\circ}$-angle with an approximate classical method (Eisner 1976). The approximate trisection of the $15^{\circ}$-angle is shown in the explanation of method $M_{8}$.

In order to construct an icosikaidigon, the $16 . \widehat{36}^{\circ}$-angle has to be constructed. If this is to be done using an astrolabe, the corresponding construction is doubly approximate and, therefore, generates a double error: the first error is due to approximate trisection of the $15^{\circ}$-angle using a compass and a straightedge (this error is stated in the first part of the analytical expressions listed in "Results" section for method $M_{6}$ ); the second error arises because each of the 21 angular differences $\Delta=\frac{\pi}{132} \mathrm{rad}=1 . \widehat{36}^{\circ}$ was determined by the sculptor based on his visual assessment (error $\varepsilon_{i}$, where $i=1 \div 21$, i.e. $i$ ranges from 1 to 21 ; his error is stated in the second part of the above-mentioned analytic expressions). In this method we assume that the errors made by the sculptor are all equal and correspond to a tenth of the angular subdivisions of the astrolabe, $\varepsilon_{i}=\frac{0.1 \pi}{180}$. The analytical equations of the angles of this method are shown in "Geometric and Statistical Control" section.

\section{Methods That We Are Not Certain Were Known in the Middle Ages}

In this subsection we describe seven approximate methods for constructing an icosikaidigon using a compass and a straightedge (Gonzalo 2001; Schneider and Sappert 1990; Virasoro 1976; Malara 1970); five of which derive from the construction of the hendecagon $\left(M_{7-11}\right)$, and the remaining two are direct methods $\left(M_{12-13}\right)$.

\section{Indirect Methods}

Here, we show two general approximate methods $\left(M_{7-8}\right)$ for constructing any regular polygon, and three specific approximate methods for constructing a hendecagon $\left(M_{9-11}\right)$. Based on these five methods, the icosikaidigon can be obtained by bisecting each of the angles.

In order to keep the paper reasonably short, we only show the methods graphically (Figs. 8, 9). With these figures, readers can get the steps to build the polygons. The analytical equations of the angles of these methods are shown in "Geometric and Statistical Control" section.

\section{Direct Methods}

Next, we show two specific methods $\left(M_{12-13}\right)$ for constructing directly the icosikaidigon. 

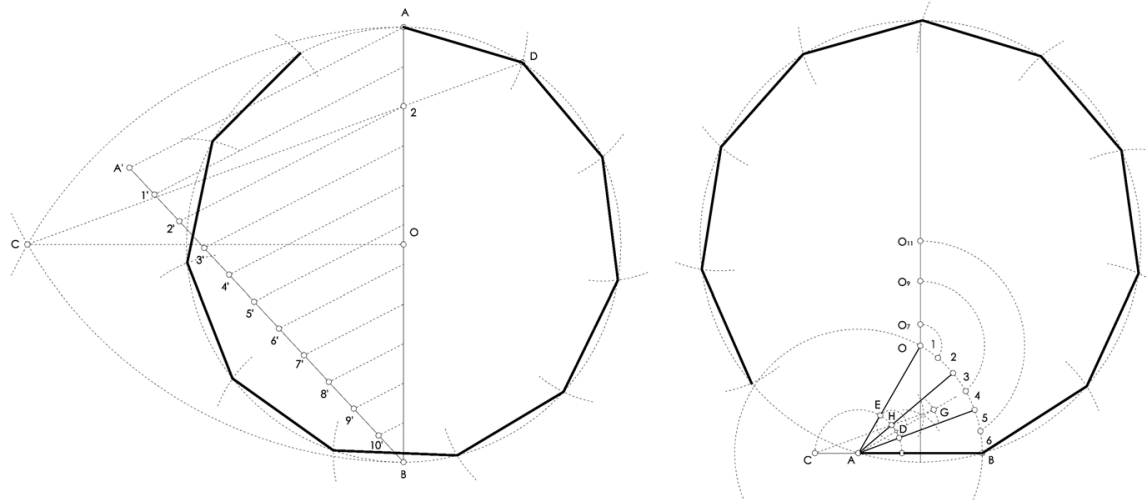

Fig. 8 On the Left, method $M_{7}$. On the right, method $M_{8}$
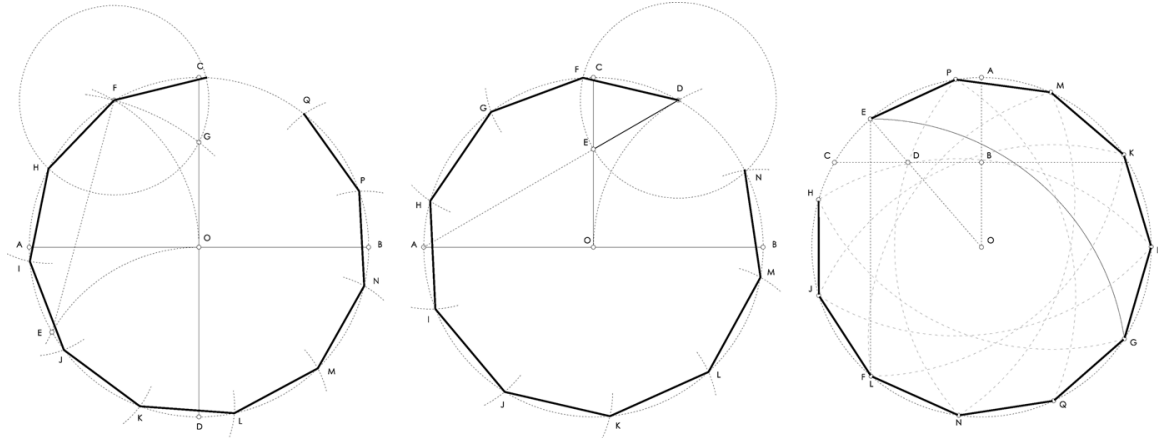

Fig. 9 Left to right: methods $M_{9}, M_{10}$ and $M_{11}$

In order to keep the paper reasonably short, we only show the methods graphically (Fig. 10). The analytical equations of the angles of these methods are shown in "Geometric and Statistical Control" section.

\section{Geometric and Statistical Control}

\section{Geometric Regression}

In addition to the foregoing, and in order to answer the research question, we must define the geometric basis for calculations. To that effect, and on the basis of the cloud of coordinated points which replicate the rose window, we assign 22 perimeter points $v_{i}$ (where $i=1 \div 22$, i.e.: $i$ ranges from 1 to 22) corresponding to the intersections of the 22 perimeter arcades (Fig. 5). Before further description of 

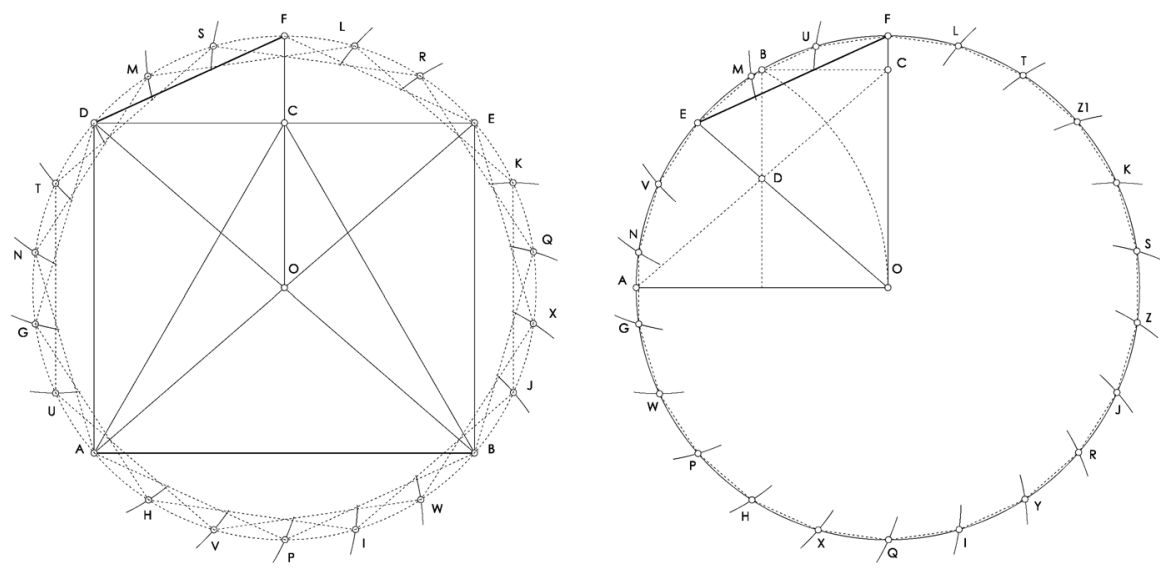

Fig. 10 On the left, method $M_{12}$; on the right, method $M_{13}$

the process, it should be noted that the maximum distance between any two points of the laser scan-obtained with a Leica ScanStation P20 scanner-is $0.0002 \mathrm{~m}$. Even though it makes little sense to work with units smaller than millimetres (especially if we are dealing with medieval techniques), we will use four decimal places for our calculations.

If we look at the positions of the 22 vertices $v_{i}$ (Fig. 5), we can see that, owing to the structural deformations and the building process itself there is no circle passing through more than three points (of these 22). For this reason, considering the positions $\left(x_{i}, y_{i}\right)$ of these 22 vertices $v_{i}$ (Table 1$)$ and using Gauss normal equations we obtain the Eq. (1) corresponding to the quadratic regression circle $\mathcal{C}$ :

$$
0.0472\left(x^{2}+y^{2}\right)-0.3484 x-0.3506 y+1=0
$$

The position of the centre of the circle $\mathcal{C}$ is $(3.6892,3.7133)$, and its radius $R=2.4937 \mathrm{~m}$.

After applying the $F$-test by Fisher-Snedecor in this circular quadratic regression, the null hypothesis $h_{0}$ of non-correlation is rejected at a significance level $\alpha=1-G(2827.54 ; 3,18)=0$. The percentage of $y$ coordinates (vertex coordinates) which are explained by the circular quadratic regression is calculated with Pearson's adjusted coefficient of determination, and it is $\eta_{a d j}^{2} \times 100=100 \%$ because $\eta_{a d j}^{2}=1-\left(1-\eta^{2}\right) \frac{22-1}{22-3-1}$, where $\eta^{2}=\frac{\sum_{i=1}^{22}\left(\hat{y}_{i}-\bar{y}\right)^{2}}{\sum_{i=1}^{22}\left(y_{i}-\bar{y}\right)^{2}}=1$ is Pearson's coefficient of determination for $\mathcal{C}$, where $\bar{y}=\sum_{i=1}^{22} \frac{y_{i}}{22}$, and $\hat{y}_{i}$ is the $y$ coordinate corresponding to the $x_{i}$ coordinate obtained as a result of the circular regression. $G\left(x ; d_{1}, d_{2}\right)=I_{\frac{d_{1} x}{d_{1} x+d_{2}}}\left(\frac{d_{1}}{2}, \frac{d_{2}}{2}\right)$ is the Fisher-Snedecor distribution, where $I$ is the regularized incomplete beta function. In other words: $\mathcal{C}$ provides a circular quadratic correlation between the coordinates of the 22 vertices of the rose window. The probability that this correlation exists is 1 , and $100 \%$ of the points' coordinates are accounted for by this correlation. Therefore, circle $\mathrm{C}$ is not only the best 
approximation but a perfect approximation, and for that reason we can consider it to be the initial circle of the rose window's real pattern.

Table 1 shows the $\left(x_{i}, y_{i}\right)$ coordinates of the 22 vertices $v_{i}$ together with the amplitudes $\varphi_{i}$ of the rose window angles $\angle\left(\overrightarrow{O v}_{i}, \overrightarrow{O v_{i+1}}\right)$, where $i=1 \div 21$ and $\varphi_{22}$ is the amplitude of angle $\angle\left(\overrightarrow{O v_{22}}, \overrightarrow{O v_{1}}\right)$. However, in order to compare the methods with the rose window, we will scale circle $\mathcal{C}$ so that its radius is $R=1 \mathrm{~m}$ (the same radius used for all methods in our analysis) and we will move the rose window so that the centre of circle $\mathcal{C}$ is the origin $(0,0)$ of the coordinate system.

\section{Geometric Comparison}

Taking into account the pattern generated by the 22 vertices $v_{i}$ and the pattern generated by each method, next we describe the geometric/statistical steps which are followed in "Method $M_{2}$ " section in order to obtain results:

1. Each of the 13 methods $M_{j}$ (where $j=1 \div 13$, i.e.: $j$ ranges from 1 to 13 ) shown in "Introduction" section generates a particular point pattern on the plane (also called $M_{j}$ ) which is made up by 22 points $\left\{u_{i, j}\right\}_{i=1}^{22}$. More specifically, in "Geometric and Statistical Control" section we present the exact analytical expressions for the coordinates of these 22 vertices $\left\{u_{i, j}\right\}_{i=1}^{22}$ for each method $\left\{M_{j}\right\}_{j=1}^{13}$.

2. For each of the vertices $v_{i}$ in Table 1 , we will rotate the rose window around point $(0,0)$ until the coordinates of that vertex are $(x, 0)$, i.e., until that vertex reaches the positive $\mathrm{x}$-semiaxis. This rotation repositions the remaining vertices and generates a particular pattern of 22 points $\left\{v_{i, k}\right\}_{k=1}^{22}$, where $k=1 \div 22$, on the plane. We will call this pattern $R_{i}$. However, since this rotation is made for each vertex $v_{i}$, we will obtain a total of 22 different point patterns $\left\{v_{i, k}\right\}_{i, k=1}^{i, k=22}$ on the plane. We will call these patterns $R_{i}$, where $i=1 \div 22$.

3. Next, after choosing the pattern $M_{j}$ generated by one of the 13 methods and choosing one of the 22 rose window patterns $R_{i}$, we will compare both geometric patterns by calculation of the following parameters:

(a) The probability $P$ of linear correlation between pattern $M_{j}$ and pattern $R_{i}$. More precisely, we will calculate the probability of rejecting the null hypothesis $h_{0}=$ "There is no linear correlation between pattern $M_{j}$ and pattern $R_{i}$ ". In order to calculate this probability $P$, we apply Student's $t$ test with $22-2$ degrees of freedom. The second column in Table 2 shows these probability values calculated for pattern $M_{1}$ and pattern $R_{i}$ (where $i$ ranges from 1 to 22), together with the resulting mean. So as not to extend this paper, for each of the following methods we will only present the respective means of parameter $P$ on the second column of Table 3 
Table 2 Parametric values corresponding to the comparison between pattern $M_{1}$ and the patterns $R_{i}$

\begin{tabular}{llll}
\hline$i$ & $P$ & $\eta_{\text {adj }}^{2} \times 100$ & $d$ \\
\hline 1 & 0.99982 & 99.96267 & 0.0037 \\
2 & 0.99978 & 99.95385 & 0.0034 \\
3 & 0.99980 & 99.95900 & 0.0032 \\
4 & 0.99981 & 99.96065 & 0.0030 \\
5 & 0.99976 & 99.94970 & 0.0030 \\
6 & 0.99990 & 99.98050 & 0.0028 \\
7 & 0.99986 & 99.97256 & 0.0029 \\
8 & 0.99979 & 99.95665 & 0.0032 \\
9 & 0.99976 & 99.95035 & 0.0035 \\
10 & 0.99971 & 99.93942 & 0.0038 \\
11 & 0.99974 & 99.94711 & 0.0041 \\
12 & 0.99973 & 99.94394 & 0.0044 \\
13 & 0.99982 & 99.96360 & 0.0047 \\
14 & 0.99975 & 99.94859 & 0.0050 \\
15 & 0.99981 & 99.96056 & 0.0051 \\
16 & 0.99972 & 99.94231 & 0.0053 \\
17 & 0.99977 & 99.95346 & 0.0052 \\
18 & 0.99977 & 99.95268 & 0.0051 \\
19 & 0.99960 & 99.91706 & 0.0051 \\
20 & 0.99964 & 99.92579 & 0.0048 \\
21 & 0.99967 & 99.93170 & 0.0045 \\
22 & 9.99961 & 0.0042 \\
Means & 0.99976 & 99.94965 & 0.0041 \\
\hline & & &
\end{tabular}

Table 3 Methods ranked in descending order according to the comparison results

\begin{tabular}{llll}
\hline Methods & $P$ & $\eta_{\text {adj }}^{2} \times 100$ & $d$ \\
\hline$M_{12}$ & 0.99996 & 99.9922 & 0.00073 \\
$M_{13}$ & 0.99995 & 99.9913 & 0.00075 \\
$M_{11}$ & 99.9895 & 0.00077 \\
$M_{4}$ & 0.99995 & 99.9808 & 0.00209 \\
$M_{3}$ & 0.99990 & 99.9745 & 0.00222 \\
$M_{8}$ & 0.99987 & 99.9735 & 0.00269 \\
$M_{6}$ & 0.99987 & 99.9554 & 0.00323 \\
$M_{1}$ & 0.99978 & 99.9496 & 0.00413 \\
$M_{5}$ & 0.99976 & 99.9438 & 0.00387 \\
$M_{9}$ & 0.99973 & 99.8907 & 0.00658 \\
$M_{7}$ & 0.99947 & 99.8141 & 0.00845 \\
$M_{2}$ & 0.99911 & 99.7642 & 0.00964 \\
$M_{10}$ & 0.99887 & 99.2966 & 0.01772 \\
\hline
\end{tabular}


(b) The extent to which one pattern $M_{j}$ accounts for the other pattern $R_{i}$. This extent is calculated by means of Pearson's adjusted coefficient of determination and Pearson's coefficient of determination. More precisely, by means of the above-mentioned correlation we will find out to what extent pattern $M_{j}$ accounts for pattern $R_{i}$. This extent is calculated by means of $\eta_{a d j}^{2} \times 100$, where $\eta_{a d j}^{2}=1-\left(1-\eta^{2}\right) \frac{22-1}{22-2-1}, \eta$ is Pearson's coefficient of determination, and $\eta_{a d j}$ is Pearson's adjusted coefficient of determination. The third column in Table 2 shows the adjusted coefficients calculated for pattern $M_{1}$ and pattern $R_{i}$ (where $i$ ranges from 1 to 22), together with the resulting mean. So as not to extend this paper, for each of the following methods we will only present the respective means of the adjusted coefficient on the third column of Table 3.

(c) The regression deviation. More precisely, we will calculate the deviation $d$ between the regression line (the straight line showing the linear correlation between pattern $M_{j}$ and pattern $R_{i}$ ) and the first and third quadrants bisector. The equation of such bisector is $y=x$, and the equation of the regression line for patterns $M_{j}$ and $R_{i}$ is $y=b+x \tan \theta$. If the architect used method $M_{j}$ to design the rose window, then the maximum deviation $d$ between both straight lines within the parameter range of the problem considered here (vertex coordinates for patterns $M_{j}$ ) must be small. More specifically, being $d=\max _{x \in[-1,1]}|b+x \tan \theta-x|$, we can see that the more similar are pattern $M_{j}$ and pattern $R_{i}$, the closer is $d$ to zero. Parameters $b$ and $\theta$ are determined using the classical means, variances and covariances calculation. The fourth column in Table 2 shows this deviation $d$ for pattern $M_{1}$ and pattern $R_{i}$ (where $i$ ranges from 1 to 22), together with the resulting mean. So as not to extend this paper, for each of the following methods we will only present the respective means of the deviation $d$ on the fourth column of Table 3 .

4. These calculations result into $13 \times 22=286$ geometric comparisons with their respective parametric values $P, \eta_{a d j}^{2} \times 100$ and $d\left(13\right.$ methods $M_{j} \times 22$ patterns $\left.R_{i}\right)$. Table 3 shows these results.

\section{Analytical Expressions and Results}

\section{Analytical Expressions of the Patterns $M_{j}$}

In this subsection we present the analytical expressions of the angles and the vertex coordinates in patterns $M_{j}$, where $j=1 \div 13$ (these are the patterns which are generated by the methods stated in "Introduction" section). 


\section{Method $M_{1}$}

If we use method $M_{1}$ for constructing a hendecagon, after bisecting each angle we obtain an icosikaidigon having 20 angles $\alpha$ plus 2 angles $(2 \pi=20 \alpha+2 \beta)$. The analytical equations of these angles are: $\alpha=\arcsin \frac{7}{25}$ and $\beta=\pi-10 \arcsin \frac{7}{25}$. The coordinates of the 22 vertices are: $u_{1,1}=(1,0) ; u_{n+1,1}=(\cos n \alpha, \sin n \alpha)$ where $n=1 \div 20$ and $u_{22,1}=(\cos (20 \alpha+\beta), \sin (20 \alpha+\beta))$.

As stated in "Approximate Methods For Constructing an Icosikaidigon" section, Table 2 shows the parameters corresponding to the comparisons between pattern $M_{1}$ and the patterns $R_{i}$, where $i=1 \div 22$.

Table 3 shows only the means of the parametric values corresponding to the comparison between pattern $M_{j}$ and the patterns $R_{i}, i=1 \div 22, j=1 \div 13$, as stated previously.

\section{Method $M_{2}$}

If we use method $M_{2}$ for constructing a hendecagon, after bisecting each angle we obtain an icosikaidigon having 20 angles $\alpha$ plus 2 angles $(2 \pi=20 \alpha+2 \beta)$. The analytical equations of these angles are: $\alpha=\arcsin \frac{2}{7}$ and $\beta=\pi-10 \arcsin \frac{2}{7}$. The coordinates of the 22 vertices are: $u_{1,2}=(1,0) ; u_{n+1,2}=(\cos n \alpha, \sin n \alpha)$ where $n=1 \div 20$ and $u_{22,2}=(\cos (20 \alpha+\beta), \sin (20 \alpha+\beta))$.

\section{Method $M_{3}$}

Method $M_{3}$ results into an icosikaidigon having 21 angles $\alpha$ plus 1 angle $\beta$, where $(2 \pi=21 \alpha+\beta)$. The analytical equations of these angles are: $\alpha=2 \arcsin \frac{1}{7}$ and $\beta=2 \pi-42 \arcsin \frac{1}{7}$. The coordinates of the 22 vertices are: $u_{1,3}=(1,0)$ and $u_{n+1,3}=(\cos n \alpha, \sin n \alpha)$, where $n=1 \div 21$.

\section{Method $M_{4}$}

If we use method $M_{4}$ for constructing a hendecagon, after bisecting each angle we obtain an icosikaidigon having 20 angles $\alpha$ plus 2 angles $\beta$, where $(2 \pi=20 \alpha+2 \beta)$. The analytical equations of these angles are: $\alpha=\frac{1}{2} \arctan \frac{16}{25}$ and $\beta=\pi-5 \arctan \frac{16}{25}$. The coordinates of the 22 vertices are: $u_{1,4}=(1,0)$; $u_{n+1,4}=(\cos n \alpha, \quad \sin n \alpha)$ where $n=1 \div 20$ and $u_{22,4}=(\cos (20 \alpha+\beta)$, $\sin (20 \alpha+\beta))$.

\section{Method $M_{5}$}

Method $M_{5}$ results into an icosikaidigon having 21 angles $\alpha$ plus 1 angle $\beta$, where $(2 \pi=21 \alpha+\beta)$. The analytical equations of these angles are: $\alpha=2 \arctan \frac{1}{7}$ and $\beta=2 \pi-42 \arctan \frac{1}{7}$. The coordinates of the 22 vertices are: $u_{1,5}=(1,0)$ and $u_{n+1,5}=(\cos n \alpha, \sin n \alpha)$, where $n=1 \div 21$. 


\section{Method $M_{6}$}

Method $M_{6}$ results into an icosikaidigon having 21 angles $\alpha_{i}$ plus 1 angle $\beta$, where $\left(2 \pi=\sum_{i=1}^{21} \alpha_{i}+\beta\right)$. The analytical equations of these angles are: $\alpha_{i}=\frac{17 \pi}{66}-$ $6 \arctan \left(\frac{2 \sin \frac{\pi}{24}}{2 \cos \frac{\pi}{24}+1}\right)+\varepsilon_{i}$ and $\beta=126 \arctan \frac{2 \sin \frac{\pi}{24}}{2 \cos \frac{\pi}{24}+1}-\frac{75 \pi}{22}-21 \varepsilon_{i}$. In these equations we have assumed that the error made by the sculptor when visually determining the angular difference $\Delta=\frac{\pi}{132} \mathrm{rad}=1 . \widehat{36}^{\circ}$ is $\varepsilon_{i}=\frac{0.1 \pi}{180}$. Therefore, the analytical expressions are as follows: $\alpha=\frac{17 \pi}{66}-6 \arctan \left(\frac{2 \sin \frac{\pi}{24}}{2 \cos \frac{\pi}{24}+1}\right)+\frac{0.1 \pi}{180}$ and $\beta=$ $126 \arctan \left(\frac{2 \sin \frac{\pi}{24}}{2 \cos \frac{\pi}{24}+1}\right)-\frac{75 \pi}{22}-21 \frac{0.1 \pi}{180}$. The coordinates of the 22 vertices are: $u_{1,5}=(1,0)$ and $u_{n+1,5}=(\cos n \alpha, \sin n \alpha)$, where $n=1 \div 21$.

\section{Method $M_{7}$}

If we use method $M_{7}$ for constructing a hendecagon, after bisecting each angle we obtain an icosikaidigon having 20 angles $\alpha$ plus 2 angles $\beta$, where $(2 \pi=20 \alpha+2 \beta)$. The analytical equations of these angles are: $\alpha=\frac{\pi}{4}-$ $\frac{1}{2}\left(\arcsin \frac{7}{2 \sqrt{103}}+\arcsin \frac{21}{2 \sqrt{309}}\right)$ and $\beta=-\frac{3}{2} \pi+5\left(\arcsin \frac{7}{2 \sqrt{103}}+\arcsin \frac{21}{2 \sqrt{309}}\right)$. The coordinates of the 22 vertices are: $u_{1,6}=(1,0) ; u_{n+1,6}=(\cos n \alpha, \sin n \alpha)$ where $n=1 \div 20$ and $u_{22,6}=(\cos (20 \alpha+\beta), \sin (20 \alpha+\beta))$.

\section{Method $M_{8}$}

If we use method $M_{8}$ for constructing a hendecagon, after bisecting each angle we obtain an icosikaidigon having 20 angles $\alpha$ plus 2 angles $\beta$, where $(2 \pi=20 \alpha+2 \beta)$. The analytical equations of these angles are: $\alpha=$ $\arctan \frac{1}{\sqrt{3}+4 \sin \left(\frac{\pi}{6}-\frac{1}{4} \arctan \frac{1}{1+\sqrt{3}}\right)}$ and $\beta=\pi-10 \arctan \frac{1}{\sqrt{3}+4 \sin \left(\frac{\pi}{6}-\frac{1}{4} \arctan \frac{1}{1+\sqrt{3}}\right)}$. The coordinates of the 22 vertices are: $u_{1,7}=(1,0) ; u_{n+1,7}=(\cos n \alpha, \sin n \alpha)$ where $n=1 \div 20$ and $u_{22,7}=(\cos (20 \alpha+\beta), \sin (20 \alpha+\beta))$.

\section{Method $M_{9}$}

If we use method $M_{9}$ for constructing a hendecagon, after bisecting each angle we obtain an icosikaidigon having 20 angles $\alpha$ plus 2 angles $\beta$, where $(2 \pi=20 \alpha+2 \beta)$. The analytical equations of these angles are: $\alpha=\arcsin \left(\sqrt{2} \sin \left(\frac{1}{2} \arcsin \frac{\sqrt{6}}{4}-\frac{1}{24} \pi\right)\right)$;

$\beta=\pi-10 \arcsin \left(\sqrt{2} \sin \left(\frac{1}{2} \arcsin \frac{\sqrt{6}}{4}-\frac{1}{24} \pi\right)\right)$. The coordinates of the 22 vertices are: $u_{1,8}=(1,0) ; \quad u_{n+1,8}=(\cos n \alpha, \quad \sin n \alpha)$ where $n=1 \div 20$ and $u_{22,8}=(\cos (20 \alpha+\beta), \sin (20 \alpha+\beta))$. 


\section{Method $M_{10}$}

If we use method $M_{10}$ for constructing a hendecagon, after bisecting each angle we obtain an icosikaidigon having 20 angles $\alpha$ plus 2 angles $\beta$, where $(2 \pi=20 \alpha+2 \beta)$. The analytical equations of these angles are: $\alpha=\arcsin \frac{\sqrt{3}}{6}$ and $\beta=\pi-10 \arcsin \frac{\sqrt{3}}{6}$. The coordinates of the 22 vertices are: $u_{1,9}=(1,0) ; u_{n+1,9}=(\cos n \alpha, \sin n \alpha)$ where $n=1 \div 20$ and $u_{22,9}=(\cos (20 \alpha+\beta), \sin (20 \alpha+\beta))$.

\section{Method $M_{11}$}

If we use method $M_{11}$ for constructing a hendecagon, after bisecting each angle we obtain an icosikaidigon having 18 angles $\alpha$ plus 4 angles $\beta$, where $(2 \pi=18 \alpha+4 \beta)$. The analytical equations of these angles are: $\alpha=$ $\arcsin \left(\frac{8 \sqrt{3}}{7} \cos \left(2 \arccos \frac{2}{\sqrt{7}}\right)\right)$ and $\beta=\frac{\pi}{2}-\frac{9}{2} \arcsin \left(\frac{8 \sqrt{3}}{7} \cos \left(2 \arccos \frac{2}{\sqrt{7}}\right)\right)$. The coordinates of the 22 vertices are: $u_{1,10}=(1,0) ; u_{n+1,10}=(\cos n \beta, \sin n \beta)$ where $n=1 \div 2 ; \quad u_{n+3,10}=(\cos (2 \beta+n \alpha), \sin (2 \beta+n \alpha)) \quad$ where $\quad n=1 \div 10$ $u_{n+11,10}=(\cos (n \beta+10 \alpha), \sin (n \beta+10 \alpha))$ where $n=3 \div 4$ and $u_{n+5,10}=(-$ $\cos (4 \beta+n \alpha), \sin (4 \beta+n \alpha))$ where $n=11 \div 17$.

\section{Method $M_{12}$}

Method $M_{12}$ results into an icosikaidigon having 14 angles $\alpha$ plus 8 angles $\beta$. The analytical equations of these angles are: $\alpha=\pi-4 \arctan \frac{\sqrt{3}}{2}$ and $\beta=-\frac{3 \pi}{2}+7 \arctan \frac{\sqrt{3}}{2}$. The coordinates of the 22 vertices are: $u_{1,11}=$ $(1,0) ; u_{n+1,11}=(\cos n \beta, \sin n \beta) \quad$ where $\quad n=1 \div 2 ; \quad u_{n+2,11}=(\cos (\beta+n \alpha)$, $\sin (\beta+n \alpha))$ where $n=2 \div 4 ; \quad u_{n+3,11}=(\cos (2 \beta+n \alpha), \sin (2 \beta+n \alpha))$ where $n=4 \div 5 ; u_{n+4,11}=(\cos (3 \beta+n \alpha), \sin (3 \beta+n \alpha))$ where $n=5 \div 7 ; u_{n+5,11}=$ $(\cos (4 \beta+n \alpha), \sin (4 \beta+n \alpha)) \quad$ where $\quad n=7 \div 9 ; \quad u_{n+6,11}=(\cos (5 \beta+n \alpha)$, $\sin (5 \beta+n \alpha))$ where $n=9 \div 11 ; u_{n+7,11}=(\cos (6 \beta+n \alpha), \sin (6 \beta+n \alpha))$ where $n=11 \div 12$ and $u_{n+8,11}=(\cos (7 \beta+n \alpha), \sin (7 \beta+n \alpha))$ where $n=12 \div 14$.

\section{Method $M_{13}$}

Method $M_{13}$ results into an icosikaidigon having 15 angles $\alpha$ plus 7 angles $\beta$. The analytical equations of these angles are: $\alpha=-\frac{3 \pi}{2}+7 \arctan \frac{\sqrt{3}}{2}$ and $\beta=\frac{7 \pi}{2}-15 \arctan \frac{\sqrt{3}}{2}$. The coordinates of the 22 vertices are: $u_{1,12}=(1,0)$; $u_{2,12}=(\cos \beta, \sin \beta) ; u_{n+2,12}=(\cos (\beta+n \alpha), \sin (\beta+n \alpha)) ; \quad$ where $\quad n=1 \div 2$; $u_{n+3,12}=(\cos (2 \beta+n \alpha), \sin (2 \beta+n \alpha))$ where $n=2 \div 4 ; u_{n+4,12}=(\cos (3 \beta+n \alpha)$, $\sin (3 \beta+n \alpha))$ where $n=4 \div 6 ; \quad u_{n+5,12}=(\cos (4 \beta+n \alpha), \sin (4 \beta+n \alpha))$ where $n=6 \div 8 ; u_{n+6,12}=(\cos (5 \beta+n \alpha), \sin (5 \beta+n \alpha))$ where $n=8 \div 10 ; u_{n+7,12}=$ $(\cos (6 \beta+n \alpha), \sin (6 \beta+n \alpha))$ where $n=10 \div 12$ and $u_{n+8,12}=(\cos (7 \beta+n \alpha)$, $\sin (7 \beta+n \alpha))$ where $n=12 \div 14$. 


\section{Results}

Table 3 below ranks the methods in descending order according to the means of their respective Pearson's adjusted coefficients of determination. From a statistical point of view, using the means only makes sense if they are very representative; this quality is measured with Pearson's coefficient of variation $C V$. As a standard, from a statistical point of view, the mean is considered to be representative if $C V<25 \%$. After making the relevant calculations with the above-mentioned parameters, we can see that all the $C V$ s obtained for the means of the adjusted coefficients $\eta_{a d j}^{2} \times$ 100 are less than $0.063 \%$. This very high degree of representativeness enables us to establish a ranking in Table 3 , which presents an optimal comparison between each pattern and the rose window. Table 3 shows the means of the parametric values corresponding to the comparison between pattern $M_{j}$ and the patterns $R_{i}$, where $i=1 \div 22$ and $j=1 \div 13$.

On the basis of the above-mentioned geometric/statistical results, Table 3 shows that the most approximate comparison is the one made with pattern $M_{12}$.

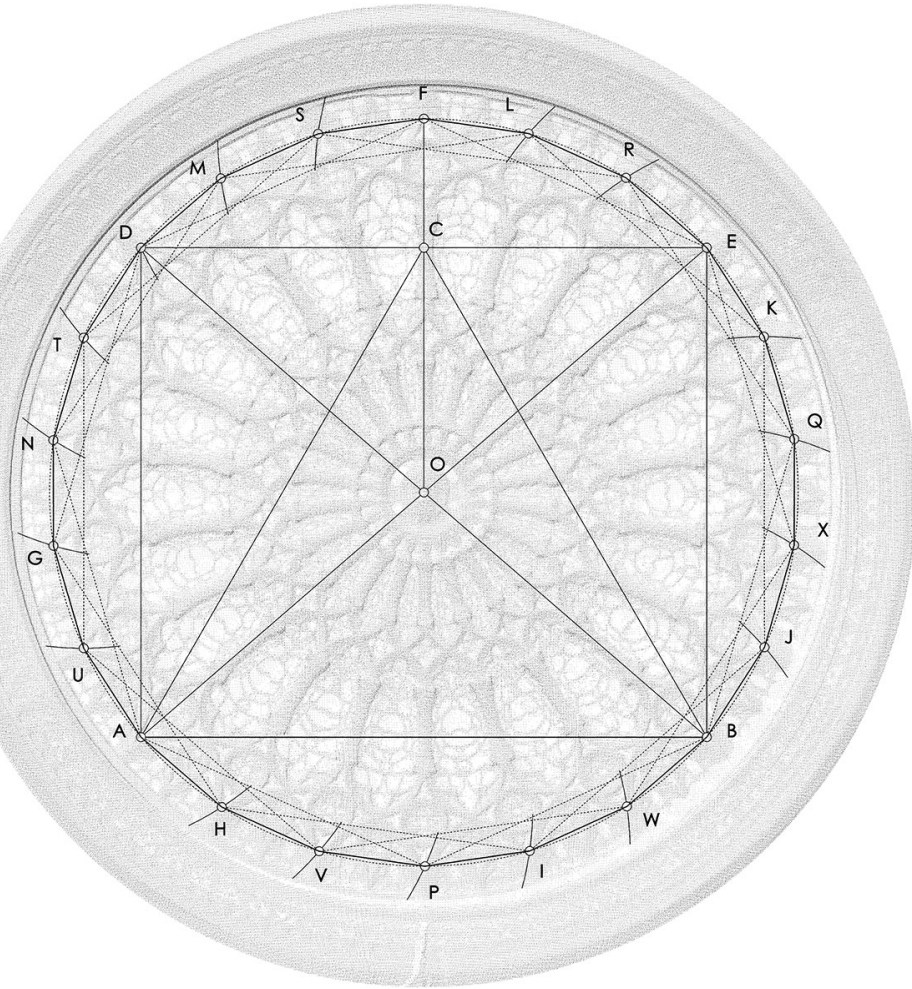

Fig. 11 Graphical result for method $M_{12}$ 


\section{Conclusion}

After this geometrically and statistically thorough analysis, we can say that method $\mathrm{M}_{12}$ is the one which best suits the design of this rose window, which is based on a 22-sided polygon.

We are certain that the methods described in "Methods That We Are Certain Were Known in the Middle Ages" section were known in the Middle Ages. On the contrary, we have not found any precise bibliographic references ensuring that the methods described in "Methods That We Are Not Certain Were Known in the Middle Ages" section were also known at that time. Therefore, if we were to choose a method from "Methods That We Are Certain Were Known in the Middle Ages" section which provides a geometric account of the rose window in Orvieto cathedral, that would be method $M_{4}$ (fourth position in the ranking of Table 3). As for the other group of methods (see "Methods That We Are Not Certain Were Known in the Middle Ages" section), in our paper we show that, despite the absence of historical records and graphic documents showing direct applications of method $M_{12}$ in medieval architecture, a stone document has survived the geometry of which mirrors almost exactly the geometry offered by method $M_{12}$ (Fig. 11). This stone document is the cathedral's rose window itself. This geometric and statistical evidence, together with the constructive simplicity of this method, leads us to claim that Andrea di Cione used method $M_{12}$ to design and build the rose window in Orvieto cathedral.

These numeric methods supplement the epistemological knowledge about the geometric techniques which were used in Gothic architecture.

Acknowledgments We would like to thank Dr. Giuseppe della Fina, Director of the Claudio Faina Museum in Orvieto; Laura Andreani, Secretary-General of the Archivio dell'Opera del Duomo di Orvieto; and the firm Leica Microsystems.

\section{References}

Baron, R. 1956. Hugonis de Sancto Victore Practica geometriae. Osiris 12: 176-224.

Bonelli, R. 1951. I disegni per la facciata del Duomo di Orvieto (1944). Bollettino dell'Istituto StoricoArtistico Orvietano 7: 1-25.

Busard, H. 1965. The Practica Geometriae of Dominicus de Clavasio. Archive for History of Exact Sciences 2 (6): 520-575.

Damiani, M. 1991. I Restauri di Giuseppe Valadier alla Facciata del Duomo di Orvieto. Architettura, Storia e documenti 1: 260-274.

Eagleton, C. 2007. Chaucer's Own Astrolabe: Text, Image and Object. Studies in History and Philosophy of Science 38 (2): 303-326.

Eisner, S. 1976. Building Chaucer's Astrolabe II. Journal of the British Astronomical Association 86: 125-132.

Gonzalo, J. 2001. Dibujo Geométrico: Arquitectura e Ingeniería. Spain: S.A. Donostiarra.

Guarnieri, A., A. Vettore and F. Remondino. 2004. Photogrammetry and Ground-Based Laser Scanning: Assessment of Metric Accuracy of the 3D Model of Pozzoveggiani Church. TS26 Positioning and Measurement Technologies and Practices II - Laser Scanning and Photogrammetry. In: The Olympic Spirit in Surveying, FIG Working Week 2004 Conference Proceedings, Greece, May 22-27.

Heath, T.L. 1908. The Thirteen Books of Euclid's Elements. Cambridge: Cambridge University Press. Lisi, F. 1997. Timaeus in Plato's Dialogues VI. Madrid: Gredos.

Malara, S. 1970. Geométrica: Disegno Geometrico. Italia: Edición Zanichelli. 
Pez, B. 1853. Hermanni Contracti monachi Augiensis de mensura astrolabii liber. (Apud R. P. Bernardum Pezium, Thesaurus Anecdotorum, tom. III, parte II, pag. 94, ex cod. ms. inclyti monasterii ad S. Petrum Salisburg. ord. S. Ben. eruto ab adm. R. et cl. D. P. Placido Bökn, ejusdem loci asceta). Patrologia Latina 143: Col. 0379 - 0390A.

Recht, R. 1989. Les Batîsseurs de Cathédrales Gothiques. Strasbourg: Musées de la ville de Strasbourg. Schneider, H. and D. Sappert. 1990. Technisches Zeichnen für die Parxis. Germany: Georg Westermann Verlag.

Shelby, L. R. 1965. Medieval Masons Tools II. Compass and Square. Technology and Culture 6 (2): 236-248.

Toldrà, J. M. et al. 2014. The Octagon, the Hendecagon and the Approximation of Pi: the Geometric Design of the Clypeus in the Enclosure of Imperial Cult in Tarraco. XII International Forum, Le vie dei Mercanti, Best Practise in Heritage Conservation Management, From the World to Pompeii. Aversa, Capri. 12-14 de Junio de 2014.

Toomer, G. J. 1984. Ptolemy's Almagest. Trans. G. J. Toomer. London: Gerald Cuckworth \& Co.

Victor, S. K. 1979. Practical Geometry in the High Middle Ages. Artis Cuiuslibet Consummatio and the Pratike de Geometrie. Philadelphia: The American Philosophical Society.

Virasoro, C. 1976. Dibujo Geométrico. Spain: Eudeba Manuales.

Wilson, J. M. 2000. Principles of Roman Architecture. 3rd edn. New Haven and London: Yale University Press.

Wu, N. 2000. Hugues Libergier and His Instruments. Nexus Network Journal 2 (4): 93-102.

Josep Lluís i Ginovart is an Architect who obtained his Ph.D. in 2002. Presently, he is a professor of intervention in architectural heritage at the Universitat Rovira i Virgili, as well as Magíster operis sedi Dertusae and Director of the Master Plan of Santa María de Tortosa Cathedral. His main fields of interest are: Medieval Architecture an Geometry.

Albert Samper is an Architect who obtained his Ph.D. in 2014. Presently, he is an associate professor of Architecture at the University Rovira i Virgili and his main fields of interest are: Fractal Geometry and the application of Geometry to Architecture.

Blas Herrera is Geometer who obtained his D.Sc. in 1994. Presently, he is a full professor of Applied Mathematics at the University Rovira i Virgili. His main fields of research interest are: Classical and Differential Geometry, and the application of Geometry to Architecture and Engineering.

Agustí Costa is an Architect who obtained his Ph.D. in 2015. Presently, he teaches Architecture at the University Rovira i Virgili andhis current research is focused on the assessment of architectural heritage and its constructive properties.

Sergio Coll is an Architect. Presently, he is Ph.D. candidate and professor of Architecture at the University Rovira i Virgili and his main fields of interest are: Architectural Heritage and the Romanesque Geometry and building. 